\title{
Bladder Outlet Obstruction as a Cause for Late Total Flap Failure in Pelvic Reconstruction with a VRAM
}

\author{
Michael J. Stein, MD ${ }^{1}$ Moein Momtazi, MSc, MD, FRCSC ${ }^{1}$ \\ ${ }^{1}$ Division of Plastic Surgery, Department of Surgery, Faculty of \\ Address for correspondence Moein Momtazi, MSc, MD, FRCSC, \\ Medicine, University of Ottawa, Ottawa, Ontario, Canada \\ The Ottawa Hospital - General Campus, Box 213, 501 Smyth Road, \\ Ottawa, Ontario, Canada K1H 8L6 (e-mail: mmomtazi@toh.on.ca).
} J Reconstr Microsurg Open 2018;3:e55-e57.

\begin{abstract}
Keywords

- pelvic reconstruction

- perineal reconstruction

- pelvic exenteration

- VRAM

- abdominoperineal resection

- flap failure

Background A 67-year-old man presented with abrupt failure of a pedicled vertical rectus abdominus myocutaneous (VRAM) flap 13 days postoperatively.

Methods The patient underwent pelvic reconstruction with a pedicled VRAM flap following sacral chordoma and abdominoperineal resection. The flap remained well perfused and viable until postoperative day 13 , at which point the patient was noted to become systemically unwell with fever, chills, and abdominal pain. This clinically coincided with prompt arterial and venous insufficiency of the VRAM flap.

Results Computed tomography of the abdomen was ordered to rule out a pelvic collection and revealed an inflated Foley catheter in the bulbar urethra. This was associated with marked distention of the bladder and bilateral hydronephrosis. Direct compression of the deep inferior epigastric pedicle by the bladder neck was noted. Conclusion The case highlights the importance of considering bladder outlet obstruction and subsequent distention as a cause of pedicle compression and VRAM flap failure following pelvic reconstruction.
\end{abstract}

Pelvic reconstruction following abdominoperineal resection and pelvic exenteration remains a significant challenge. Perineal wound complications occur in up to $60 \%{ }^{1-3}$ of patients with primary closure. The high morbidity associated with primary closure is attributable to the large pelvic dead space, which can lead to fluid collection and superinfection, the high tension skin closure, and the presence of irradiated and poorly vascularized tissues. ${ }^{4-6}$ The vertical rectus abdominus myocutaneous (VRAM) flap has emerged as the workhorse flap in pelvic reconstruction, associated with significant reductions in perineal morbidity compared to primary closure alone. ${ }^{7}$ The advantages of flap reconstruction in this context are the obliteration of pelvic dead space, interposition of well-vascularized tissue into an irradiated wound bed, a decrease in closure tension, and resistance of infection. ${ }^{8-12}$ Despite the purported benefits, the VRAM flap is associated with the inherent risks of any pedicled flap reconstruction, namely, donor site morbidity and the risk of partial or complete flap failure. The rate of complete flap loss following VRAM-based pelvic reconstruction is less than
$5 \%,{ }^{13-17}$ with the vast majority of failures occurring within the first 72 hours. The rates and etiology of late flap failure, or those occurring after postoperative day 7, however, are poorly elucidated. Herein, we propose one such mechanism of late flap failure, occurring 13 days postoperatively due to pedicle compression secondary to bladder distension. To the best of our knowledge, this is the first reported case of VRAM flap loss via this mechanism.

\section{Case Report}

A 67-year-old man with a history of multiple cerebrovascular events, a myocardial infarction, hypertension, and a 30 packyear smoking history presented with dull 5/10 sacral pain, prompting a pelvic magnetic resonance imaging (MRI) examination. Imaging revealed a large, well circumscribed, heterogeneously enhancing mass through the mid body of the sacrum. Subsequent core biopsy demonstrated a sacral chordoma.

The patient underwent preoperative external beam radiation, followed by sacrectomy and abdominoperineal resection. received

June 6, 2018

accepted

July 25,2018
DOI https://doi.org/

10.1055/s-0038-1669453. ISSN 2377-0813.
Copyright $\odot 2018$ by Thieme Medical Publishers, Inc., 333 Seventh Avenue, New York, NY 10001, USA. Tel: +1(212) 584-4662.
License terms

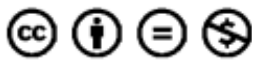




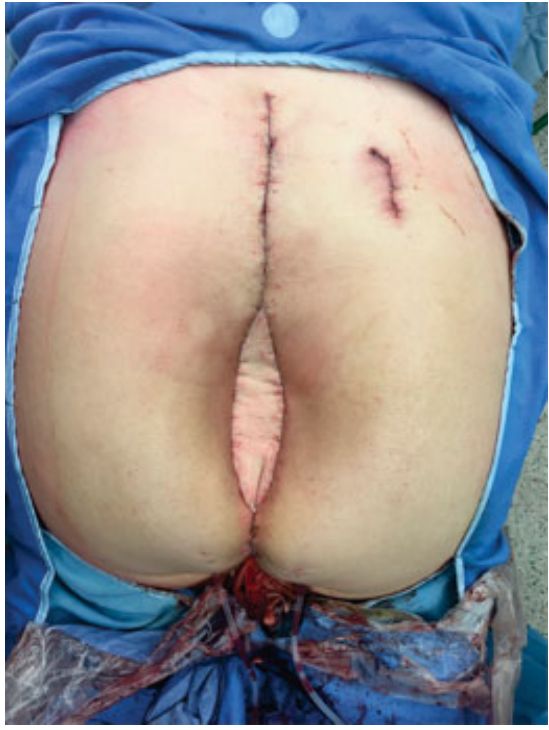

Fig. 1 Intraoperative photograph.

The colostomy was exteriorized through the left rectus abdominus muscle. The resultant pelvic and perineal defect was reconstructed using a right VRAM flap (-Fig. 1).

The patient did well postoperatively. Following an unremarkable 5-day admission to the intensive care unit, the

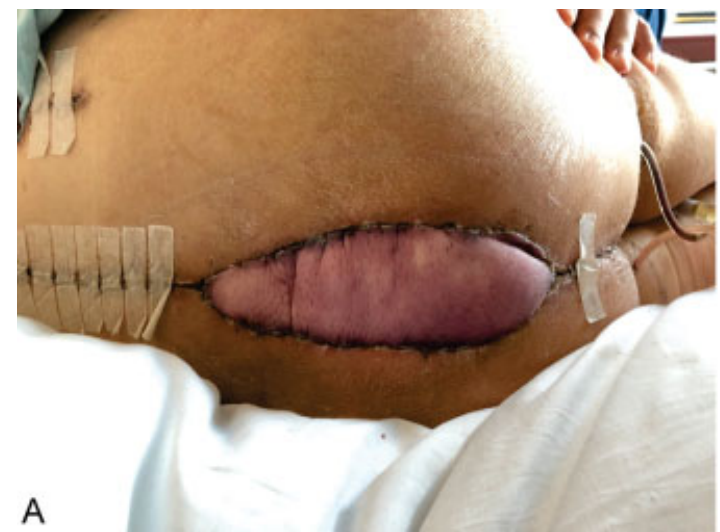

\section{A}

Fig. 2 (A) Postoperative day 13. (B) Postoperative day 14. patient was transferred to the orthopedic surgery unit. Regular flap checks continued to be performed and the flap was consistently well perfused. On postoperative day 13, the patient became systemically unwell with fever, tachycardia, and significant abdominal discomfort. Laboratory investigations demonstrated an increase in white blood cell count and a septic workup was initiated. During this time, the flap demonstrated signs of both venous and arterial insufficiency (-Fig. 2). Computed tomography of the pelvis was ordered to assess for intrapelvic collection and revealed a markedly distended bladder with bilateral hydronephrosis. The deep inferior epigastric pedicle could be visualized and was noted to be occluded by the distended bladder, preventing arterial inflow and venous outflow (-Fig. 3). The bladder was promptly decompressed, resulting in improvement in the patient's clinical status. The perfusion to the flap, however, did not improve, ultimately resulting in total flap loss.

The patient was brought back to the operating room 3 weeks postoperatively for debridement of the VRAM flap. Significant thrombosis of the deep inferior epigastric pedicle could be appreciated in the context of a frankly necrotic flap. Following flap debridement, bilateral advancement flaps were elevated for closure. The patient was discharged from hospital postoperative day 40 and the wound was completely healed by postoperative day 60 .

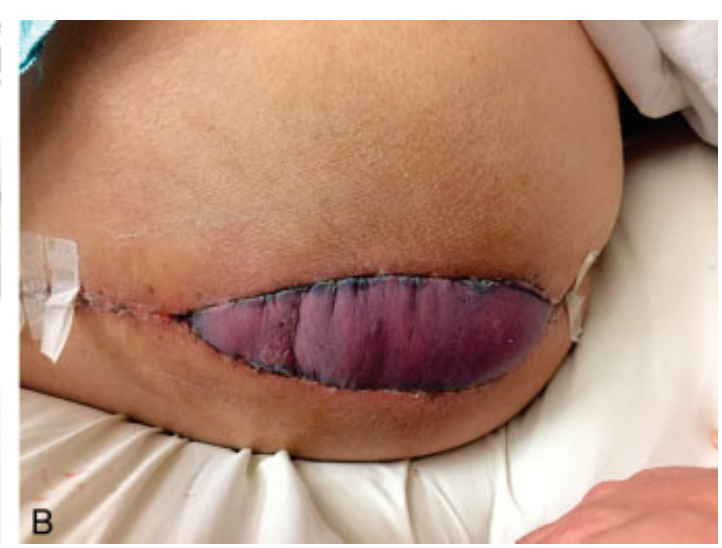

B

,
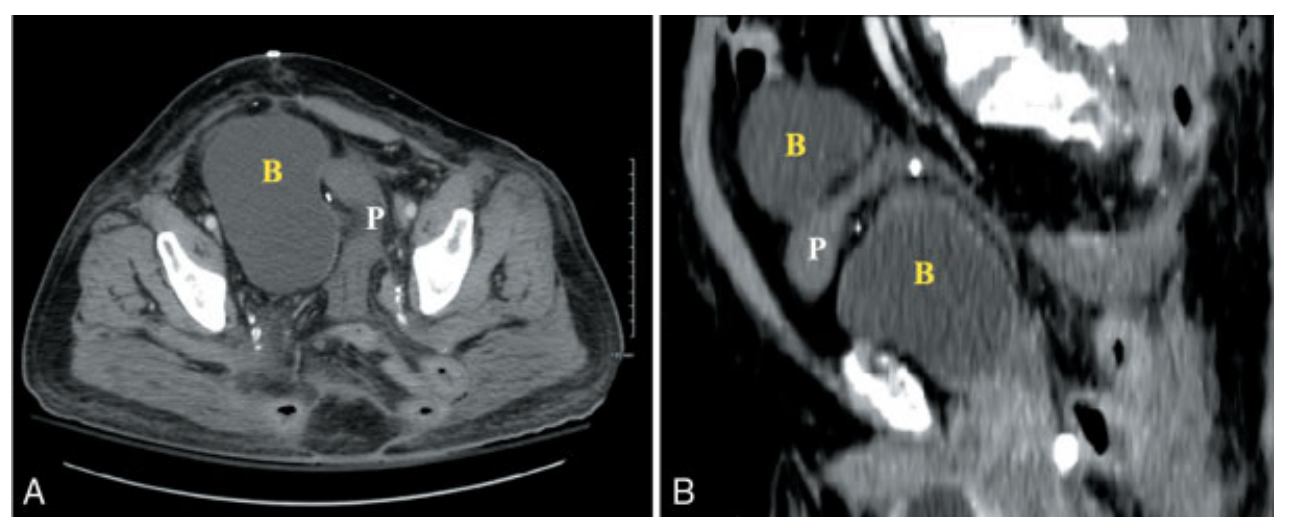

Fig. 3 (A and B) Computed tomography of pelvis showing markedly distended bladder compressing VRAM pedicle. VRAM, vertical rectus abdominus myocutaneous. 


\section{Discussion}

Flap failure in either free or pedicled flap reconstruction typically occurs within the first 72 hours. This is often the result of intrinsic pedicle thrombosis (arterial or venous) or extrinsic compression, eventually leading to thrombosis. In the immediate postoperative period, traction or kinking of the pedicle is the most likely culprit for flap failure. The incidence of and mechanisms for late flap failure, however, remain poorly understood. The described case demonstrates clearly that pedicle compression from a distended bladder is a potential mechanism for late flap failure and should be considered on the differential when assessing a flap with clinical signs of vascular compromise. Furthermore, it illustrates the utility of cross-sectional imaging in helping to elucidate the mechanisms of late flap failure due to pedicle compression.

\section{Funding \\ None. \\ Conflict of Interest \\ None.}

\section{References}

1 Touran T, Frost DB, O'Connell TX. Sacral resection. Operative technique and outcome. Arch Surg 1990;125(07):911-913

2 McAllister E, Wells K, Chaet M, Norman J, Cruse W. Perineal reconstruction after surgical extirpation of pelvic malignancies using the transpelvic transverse rectus abdominal myocutaneous flap. Ann Surg Oncol 1994;1(02):164-168

3 Khoo AK, Skibber JM, Nabawi AS, et al. Indications for immediate tissue transfer for soft tissue reconstruction in visceral pelvic surgery. Surgery 2001;130(03):463-469

4 Mathes SJ, Feng LJ, Hunt TK. Coverage of the infected wound. Ann Surg 1983;198(04):420-429

5 Baird WL, Hester TR, Nahai F, Bostwick J III. Management of perineal wounds following abdominoperineal resection with inferior gluteal flaps. Arch Surg 1990;125(11):1486-1489
6 Anthony JP, Mathes SJ. The recalcitrant perineal wound after rectal extirpation. Applications of muscle flap closure. Arch Surg 1990;125(10):1371-1376

7 Devulapalli C, Jia Wei AT, DiBiagio JR, et al. Primary versus flap closure of perineal defects following oncologic resection: a systematic review and meta-analysis. Plast Reconstr Surg 2016;137 (05):1602-1613

8 Ferenschild FT, Vermaas M, Hofer SO, Verhoef C, Eggermont AM, de Wilt JH. Salvage abdominoperineal resection and perineal wound healing in local recurrent or persistent anal cancer. World J Surg 2005;29(11):1452-1457

9 Galandiuk S, Jorden J, Mahid S, McCafferty MH, Tobin G. The use of tissue flaps as an adjunct to pelvic surgery. Am J Surg 2005;190 (02):186-190

10 Jeon H, Yoon ES, You HJ, Kim HS, Lee BI, Park SH. Comparison of the complications in vertical rectus abdominis musculocutaneous flap with non-reconstructed cases after pelvic exenteration. Arch Plast Surg 2014;41(06):722-727

11 Butler CE, Gundeslioglu AO, Rodriguez-Bigas MA. Outcomes of immediate VRAM flap reconstruction of irradiated abdominoperineal resection defects. J Am Coll Surg 2008;206:694-703

12 Radice E, Nelson H, Mercill S, Farouk R, Petty P, Gunderson L. Primary myocutaneous flap closure following resection of locally advanced pelvic malignancies. Br J Surg 1999;86(03):349-354

13 Buchel EW, Finical S, Johnson C. Pelvic reconstruction using vertical rectus abdominis musculocutaneous flaps. Ann Plast Surg 2004;52(01):22-26

14 Bakx R, van Lanschot JJ, Zoetmulder FA. Inferiorly based rectus abdominis myocutaneous flaps in surgical oncology: indications, technique, and experience in 37 patients. J Surg Oncol 2004;85 (02):93-97

15 Horch RE, Hohenberger W, Eweida A, et al. A hundred patients with vertical rectus abdominis myocutaneous (VRAM) flap for pelvic reconstruction after total pelvic exenteration. Int J Colorectal Dis 2014;29(07):813-823

16 Chessin DB, Hartley J, Cohen AM, et al. Rectus flap reconstruction decreases perineal wound complications after pelvic chemoradiation and surgery: a cohort study. Ann Surg Oncol 2005;12(02): 104-110

17 Sunesen KG, Buntzen S, Tei T, Lindegaard JC, Nørgaard M, Laurberg S. Perineal healing and survival after anal cancer salvage surgery: 10-year experience with primary perineal reconstruction using the vertical rectus abdominis myocutaneous (VRAM) flap. Ann Surg Oncol 2009;16(01):68-77 